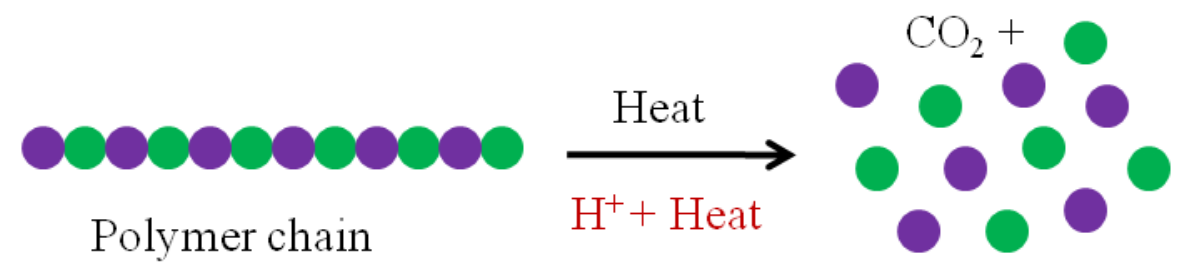

Vaporizable organics

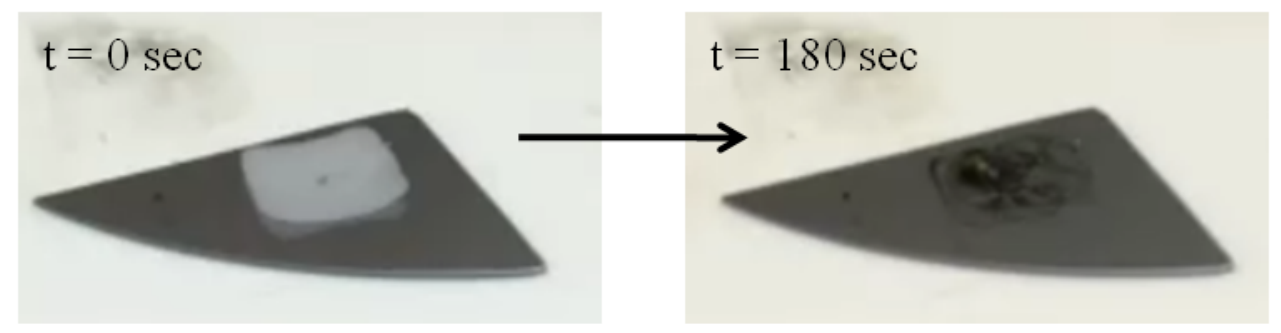

(C) 2016. This manuscript version is made available under the Elsevier user license http://www.elsevier.com/open-access/userlicense/1.0/ 


\title{
Transient Materials from Thermally-Sensitive Polycarbonates and Polycarbonate Nanocomposites
}

Katherine L. Camera ${ }^{1}$, Brandon Wenning ${ }^{1}$, Amit Lal ${ }^{2}$, and Christopher K. Ober ${ }^{3 *}$

${ }^{1}$ Department of Chemistry and Chemical Biology, Cornell University, Ithaca, NY 14853.

${ }^{2}$ Department of Electrical and Computer Engineering, Cornell University, Ithaca, NY 14853.

${ }^{3}$ Department of Materials Science and Engineering, Cornell University, Ithaca, NY 14853.

*Corresponding Author: email: cko3@cornell.edu, address: 310 Bard Hall, Cornell University, Ithaca, NY 14853

Keywords: Polycarbonates, thermal degradation, acid catalysis, Young's modulus, OMMT

\begin{abstract}
:
Transient materials, or materials with designed short lifetimes, are of great interest in many fields of research. This work describes the development of polycarbonates as a thermallytriggered vaporizable substrate platform. Good mechanical properties over operational temperature ranges in combination with fast and clean decomposition at slightly higher temperatures create an effective transient substrate. Therefore, characterization of the thermal and mechanical properties of vaporizable polycarbonates (VPC) and their composites are indispensable for creating such materials systems. A tailor-made VPC and its blends with a commercially available poly(propylene carbonate) were investigated. Both polycarbonates have acid sensitive backbones; therefore, when combined with an acid source, catalyzed decomposition was observed within minutes, achieving a range of decomposition temperatures between $160{ }^{\circ} \mathrm{C}$ and $250{ }^{\circ} \mathrm{C}$. Polymer with organo-modified montmorillonite nanocomposites were investigated to provide structural support. These nanocomposites resulted in Young's moduli ranging from $4 \mathrm{MPa}$ to $\sim 2 \mathrm{GPa}$. Their tunable nature allows for designer control over the substrate's properties based on application needs.
\end{abstract}




\section{Introduction:}

Transient polymeric materials are designed with a short or temporary lifetime and are attracting much interest in various fields of research. A controlled or triggerable loss of the physical and/or functional properties of these materials has found use in applications including fabrication of nano- or microchannels in fluidics devices ${ }^{1-5}$, controlled drug release ${ }^{6-10}$, and transient electronics ${ }^{11-17}$. In particular, the concept of a fully transient electronic device has gained momentum for technological advancement and potential applications where collection, recovery, or disposal of the material may be difficult. To create an effective transient electronic device, it must be accompanied by transient substrates for support, and different applications will require specifically tailored properties (e.g. chemical, mechanical, and physical) from these transient materials. Recent work has shown that use of biodegradable polymers and electronic components can be combined to achieve a fully degradable implant for biomedical devices. Transient electronic components have been developed and circuits were tested on multiple biodegradable substrates including poly(lactic-co-glycolic acid), poly(lactic acid), poly(caprolactone), rice paper, and silk, where water and phosphate buffer solution are used to dissolve the substrate and electronic components ${ }^{11,13,14,16}$. Another study by Acar et al. showed that poly(vinyl alcohol) composites with glucose and sucrose could be fabricated into transient substrates with a triggered degradation occurring in the presence of water ${ }^{15}$. However, solution triggered degradation of these polymers limits their applications. Moore and coworkers have used metastable cyclic poly(phthalaldehyde) (cPPA) as a UV-sensitive substrate material for printed electronics ${ }^{12}$. When cPPA is combined with a photoacid generator (PAG) and cast into a substrate, UV light can be used to activate the PAG, which releases acid, which in turn causes chain unzipping (degradation) of cPPA. Upon continuous exposure to UV light there was destruction of a free standing transistor array on cPPA, leaving behind a slight residue. Park et. al. also showed that cPPA can be thermally triggered to degrade in the presence of acid, where heat is used to release encapsulated acid that degrades both the cPPA and the electronic components ${ }^{17}$. However, in both cases some material remains.

In this paper, we report a thermally-sensitive polycarbonate system as a vaporizable transient

substrate with tunable thermal and mechanical properties. Polycarbonates, like the polyphthalaldehydes, are acid sensitive, allowing for catalyzed decomposition in the presence of 
acid and heat. Polycarbonates can be designed to degrade into volatile compounds, leaving no substantial residue behind upon disintegration, allowing for effective substrate transience. Previous work in the field of lithography has shown effective vaporization of polycarbonates when subject to acid and heat ${ }^{18-20}$. Applying these techniques, thin and thick film substrates containing acid can be heated leading to clean and fast decomposition. Two polycarbonates, poly(propylene carbonate) and a synthesized vaporizable polycarbonate (VPC) were investigated separately, and in a blend of the two as thermally-sensitive substrate materials. Studies of the thermal degradation of PPC indicate that the decomposition of PPC occurs via chain unzipping and chain scission, producing volatile products of cyclic propylene carbonate and 1,2propanediol, and that the addition of certain PAGs can significantly reduce the degradation temperature ${ }^{21,22}$. The thermal degradation products of polycarbonates, similar to the VPC synthesized in this report, were studied and found to also be volatile ${ }^{23}$. In this study we confirm the use of PAGs for acid catalyzed degradation and introduce a new acid source, a thermal acid generator (TAG), which also effectively catalyzes degradation of these polycarbonates and their combination. The use of multiple acid sources allows for tunable degradation temperatures. Lowering the degradation temperature, for example, lowers the amount of energy needed to vaporize a polymer, making transience more facile.

Thermally-triggered transience is not the only important property needed in a substrate. To be an effective substrate or packaging for transient electronics, the material must have sufficient mechanical strength in order to allow the device to function during its operational lifetime. Different applications require various mechanical properties in substrates. Therefore, the modulus, toughness, and tensile strength of the polycarbonates were assessed and nanocomposites with an organo-modified montmorillonite (OMMT) (clay) were investigated as a reinforcing phase to give a range of mechanical properties. The ability to behave as a stable substrate during process and operating conditions, possessing tunable thermal and mechanical properties, and employ of a thermal trigger for substrate degradation allow this polycarbonate system to achieve criteria necessary to be an effective transient substrate. 


\section{Experimental:}

Materials

Reagents for the synthesis of VPC were purchased from Sigma Aldrich and used without purification, except for tetrahydrofuran, which was dried over calcium hydride before use. Triphenylsulfonium triflate was used as photoacid generator 1 (PAG1) and was purchased from Sigma Aldrich and used without further purification, and P-cumenyl(p-tolyl)iodonium tetrakis(pentafluorophenyl)borate was used as PAG2 and was purchased from Boc Sciences and used without further purification. 2,4,5-trichloronbenzenesulfonic acid was used as a thermal acid generator (TAG) and was purchased from Sigma Aldrich and used without further purification. Organo-modified montmorillonite (OMMT) 20A was purchased from Southern Clay Products. Poly(propylene carbonate) (PPC) at $130 \mathrm{kDa}$ was supplied by Novomer.

\section{Characterization}

Molecular weight of VPC was determined using gel permeation chromatography (GPC) using a Waters Ambient Temperature GPC with THF as the solvent and measured against a polystyrene standard. The decomposition temperature $\left(\mathrm{T}_{\mathrm{d}}\right)$ was measured on a TA Instruments Q500 Thermogravimetric Analyzer (TGA) with a $10{ }^{\circ} \mathrm{C} / \mathrm{min}$ ramp rate. The thermal decomposition products from the polymers were analyzed using gas chromatography mass spectrometry (GC-MS) with an Agilent 6890 GC equipped with an autosampler and a JEOL double-focusing sector MS. High resolution images were taken using a Tescan-Mira3 scanning electron microscope (SEM). Young's modulus for samples was measured on TA Instruments Q800 Dynamic Mechanical Analyzer (DMA) using a constant force ramp of $1 \mathrm{~N} / \mathrm{min}$ at room temperature. Film thickness was measured using a P10 profilometer.

\section{Synthesis of VPC}

The custom-made vaporizable polycarbonate (VPC) was synthesized according to previous procedures ${ }^{19,23}$, and the final structure is depicted in Figure 1 (along with structures of PPC, PAG1, PAG2, and TAG). VPC had a molecular weight of $3.5 \mathrm{kDa}$. 


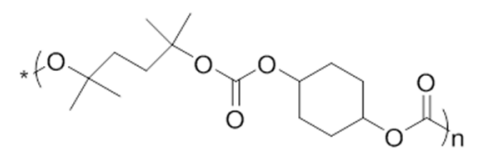

$\mathrm{VPC}$

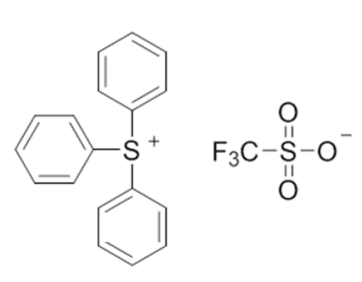

PAG1

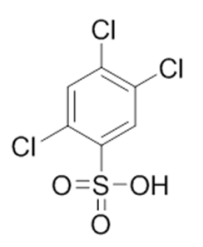

TAG

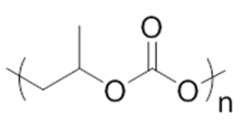

PPC
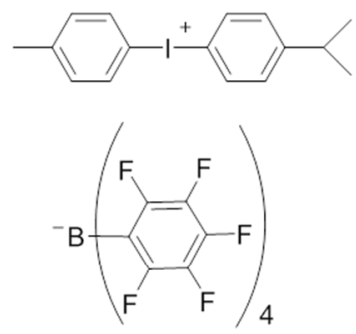

PAG2

Figure 1. Chemical Structures of compounds used in this study.

Film Formation

Solutions of VPC, VPC + PAG, and VPC + TAG were made by dissolving $5 \mathrm{wt} \%$ of VPC in dichloromethane (DCM) and adding in $5 \mathrm{wt} \%$, with respect to solid content, of either PAG1 or TAG (or neither in the case of the native VPC solution). The solution was stirred vigorously to ensure complete dissolution and dispersion in DCM. The stirred solution was then dropped casted onto a silicon wafer and spun at a rate of $2000 \mathrm{rpms}$ for $60 \mathrm{~s}$ with a ramp rate of $1000 \mathrm{rpms} / \mathrm{s}$. The spin coated film was then subjected to a post apply bake for $60 \mathrm{~s}$ at $120{ }^{\circ} \mathrm{C}$.

Solutions of PPC, PPC + PAG2, and PPC + TAG were made by dissolving $10 \mathrm{wt} \%$ of PPC in DCM and adding in $5 \mathrm{wt} \%$, with respect to solid content, of either PAG2 or TAG (or neither in the case of the native PPC solution). The solution was stirred vigorously to ensure complete dissolution and dispersion in DCM. The stirred solution was then casted onto PDMS substrate and allowed to dry at room temperature overnight.

PPC and VPC were also mixed in solution to create a PPC/VPC blend. PPC and VPC were mixed in a 70/30 ratio respectively in DCM to make the blend. Solutions of the blend + PAG2 and blend + TAG were made by dissolving $5 \mathrm{wt} \%$ with respect to solid content of the 
PAG2 or TAG into the blend in DCM. The solutions were stirred vigorously and subsequently casted onto a PDMS substrate and allowed to dry at room temperature overnight.

Composites of PPC and the PPC/VPC blend with OMMT were made according to a previously published procedure with some modifications ${ }^{24}$. First a $10 \mathrm{wt} \%$ solution of PPC (or PPC/VPC blend) in DCM was made and sonicated until the polymer was completely dissolved. Next a separate solution of OMMT was made by mixing in 2,5 , or $10 \mathrm{wt} \%$ of the respective additive (the weight percent is with respect to the total solid polymer content) in DCM and sonicating the mixture at $2 \mathrm{~W}$ for 3 min using a cell disruptor sonication probe. These sonicated mixtures were then rapidly stirred in a flask for $2 \mathrm{hrs}$. After $2 \mathrm{hrs}$, the polymer solution and the stirred mixture were combined and sonicated at $2 \mathrm{~W}$ for $3 \mathrm{~min}$. Then polymer composite solution was placed in a flask and heated and stirred at $40{ }^{\circ} \mathrm{C}$ for $2 \mathrm{hrs}$. The polymer composites were then solution casted onto a PDMS substrate and allowed to dry at room temperature overnight.

\section{DUV Exposure}

Films (after casting/drying) with PAG (1 or 2) were exposed to deep ultraviolet (DUV) light in order to activate the PAG. Films containing PAG1 were exposed to a dose of $100 \mathrm{~mJ} / \mathrm{cm}^{2}$ and films containing PAG2 were exposed to a dose of $4 \mathrm{~J} / \mathrm{cm}^{2}$ using a hand-held UV lamp at a $254 \mathrm{~nm}$ wavelength.

\section{Results and Discussion:}

\section{Film Formation}

Solution based casting techniques were used due to the thermal sensitivity of the polymers. By investigating polymers that are designed to decompose at lower temperatures, molding techniques such as melt processing may not be viable and low temperature casting methods, such as spin coating and solution casting, were used. Spin coating of VPC, VPC + PAG1, and VPC + TAG resulted in homogeneous thin films with a thickness of $150 \mathrm{~nm}$. Solution casting of a $10 \mathrm{wt} \%$ VPC solution in DCM was attempted, but resulted in a brittle, flakey film not suitable for thickness or mechanical characterization. This is not unexpected, as the molecular weight of the VPC is low $(3.5 \mathrm{kDa})$ and below chain entanglement which would lead to poor thick film properties. Solution casting of PPC, PPC + PAG2, and PPC + TAG, the 
corresponding blend solutions, and the PPC and blend composites resulted in thicker films of approximately $150 \mu \mathrm{m}$, and could easily be peeled off the PDMS substrate to give free-standing films. As the PPC has a much higher molecular weight (130 kDa) than the VPC, it alone can form stable thick films and it is added to VPC to provide ductility and form stable thick films of this blended polymer. The thickness of the film can be controlled, and films ranging from $100-$ $300 \mu \mathrm{m}$ were achieved. Spin coating of PPC films was done using a $5 \mathrm{wt} \%$ solution of PPC in DCM and followed the same spin coating procedure as for VPC. Spin coating of PPC resulted in films with a thickness of $5 \mu \mathrm{m}$. By changing the film preparation we were able to achieve films of varying thickness which can be applied to numerous applications.

\section{Thermal Analysis}

In order to create an effective thermally-sensitive substrate for transient materials, the polymer should be stable during fabrication and operation and only degrade upon introduction of a thermal trigger. Since many polymers are created to withstand high temperatures and not decompose, alternative and metastable polymers must be considered for transient material application. Polycarbonates have high decomposition temperatures, but they have acid sensitive backbones; therefore, when blended with an acid source, catalyzed decomposition is observed, allowing for the potential to reach lower decomposition temperatures that are just above operational temperatures. Multiple acid catalysts were screened to determine the effect on VPC, PPC, and the blend, by investigating degradation temperatures and thermal profiles for all casted films using thermogravimetric analysis (TGA). From previous literature, PAG1 and PAG2 were determined to be the most effective PAGs for the respective polymers, and PAG2 was used in the blend system since the majority component was PPC $^{18,22}$. Multiple compounds were screened as potential TAGs to effectively catalyze the decomposition of both polymers, and 2,4,5trichlorobenzenesulfonic acid was determined to be the best candidate.

Spin coated films of VPC, VPC + PAG1, and VPC + TAG were scraped off their wafer substrate and the resulting powder was analyzed. The onset degradation temperature $\left(\mathrm{T}_{\mathrm{d} \text {,onset }}\right)$ and the degradation temperature at 50\% weight loss $\left(\mathrm{T}_{\mathrm{d}, 50}\right)$ can be seen in Figure 2, along with the thermal profiles during the heating ramp for all three samples. As expected, the acid sources decrease the decomposition temperature, with the addition of $5 \mathrm{wt} \%$ PAG1 lowering the $\mathrm{T}_{\mathrm{d}, 50}$ by $80{ }^{\circ} \mathrm{C}$ and the addition of $5 \mathrm{wt} \%$ TAG lowering the $\mathrm{T}_{\mathrm{d}, 50}$ by $98{ }^{\circ} \mathrm{C}$. Solution cast films of PPC, 
$\mathrm{PPC}+\mathrm{PAG} 2$, and PPC + TAG were peeled from their PDMS substrate and a piece of each film was analyzed. The $T_{d}$,onset and $T_{d}, 50$ can been seen in Figure 2. As with VPC, the addition of acid sources to PPC lowered the $\mathrm{T}_{\mathrm{d}, 50}$ from $255{ }^{\circ} \mathrm{C}$ to $136{ }^{\circ} \mathrm{C}$ and $146{ }^{\circ} \mathrm{C}$ with PAG2 and TAG, respectively. The polymer blend material (70/30 PPC/VPC) was also subjected to thermal analysis as well as its acid composites. The $\mathrm{T}_{\mathrm{d} \text {,onset }}$ and $\mathrm{T}_{\mathrm{d}, 50}$ can been seen in Figure 2. Like its individual polycarbonate components, the blend showed a decrease in decomposition temperature with the addition of PAG2 and TAG. The blend had an initial $\mathrm{T}_{\mathrm{d}, 50}$ of $233^{\circ} \mathrm{C}$, and PAG2 and TAG were able to catalyze decomposition and lower the $\mathrm{T}_{\mathrm{d}, 50}$ to $208{ }^{\circ} \mathrm{C}$ and $165^{\circ} \mathrm{C}$, respectively. All of the polymer systems investigated exhibited thermal decomposition that leaves less than $5 \mathrm{wt} \%$ material remaining after heating, meaning there is very little residue (if any) left behind. These systems show that the acid catalysts are effective for both the individual polycarbonates as well as a blend of the two. By catalyzing the decomposition reaction of the polymers, less thermal energy is required to decompose them, allowing for thermal degradation to be more easily achieved. The use of different acids also allows for a variety of decomposition temperatures to be reached, which gives way to a tunable system based on application needs.
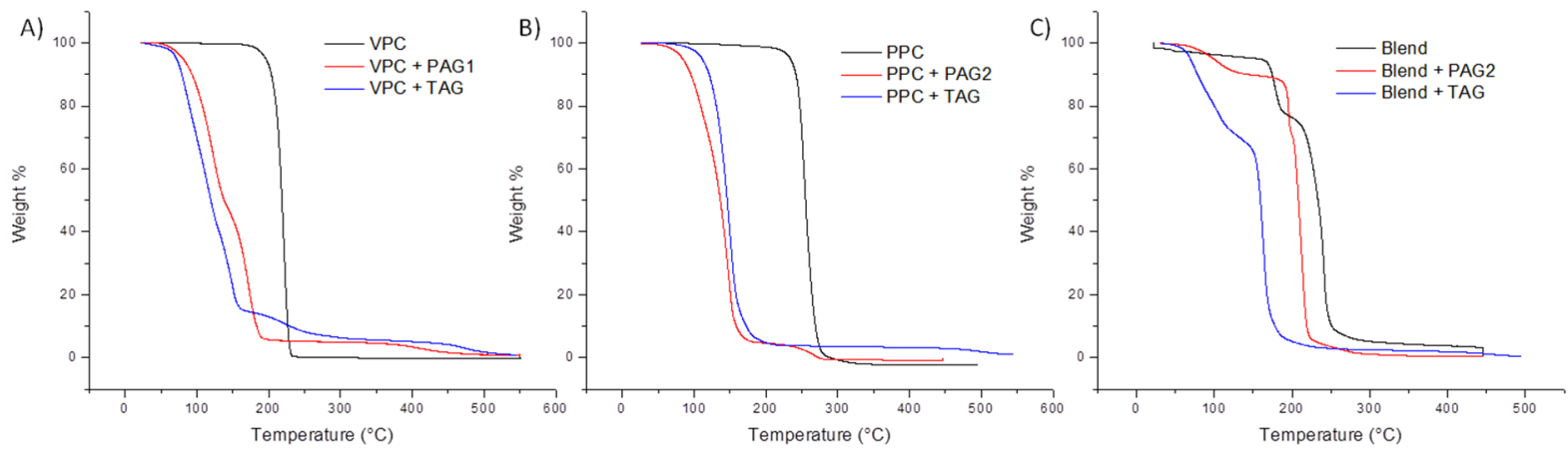

\begin{tabular}{|c|c|c|c|c|c|c|c|c|c|}
\hline Sample & VPC & $\begin{array}{l}\text { VPC }+ \\
\text { PAG1 }\end{array}$ & $V P C+T A G$ & PPC & $\begin{array}{l}\text { PPC + } \\
\text { PAG2 }\end{array}$ & $\mathrm{PPC}+\mathrm{TAG}$ & Blend & $\begin{array}{l}\text { Blend + } \\
\text { PAG2 }\end{array}$ & $\begin{array}{c}\text { Blend + } \\
\text { TAG }\end{array}$ \\
\hline $\begin{array}{l}\text { Onset } T_{d} \\
\left({ }^{\circ} \mathrm{C}\right)\end{array}$ & 197 & 70 & 66 & 224 & 79 & 109 & 178 & 106 & 62 \\
\hline $\begin{array}{c}50 w t \% T_{d} \\
\left({ }^{\circ} \mathrm{C}\right)\end{array}$ & 218 & 138 & 120 & 255 & 136 & 146 & 233 & 208 & 165 \\
\hline
\end{tabular}

Figure 2. Thermal Analysis of VPC with and without acid additives (A), PPC with and without acid additives (B), the 70/30 PPC/VPC blend with and without acid additives, and the $\mathrm{T}_{\mathrm{d}, \text { onset }}$ and $\mathrm{T}_{\mathrm{d}, 50}$ for each (D). 
Since the TAG has a free acid group, the room temperature stability over time of the acid-sensitive polycarbonates blended with TAG was measured. Film stability at room temperature was tested using the 70/30 PPC/VPC blend + TAG. Five films were cast as described above and were placed in glass vials and left at room temperature. After a designated amount of time, the film was removed and subjected to thermal analysis using TGA and the change in decomposition over time was monitored. As seen in Figure 3, films were tested over a 7 week period and their thermal degradation profiles and degradation temperatures at $50 \mathrm{wt} \%$ loss were compared. The degradation temperature remained the same for all samples from 1 day to 7 weeks at $170{ }^{\circ} \mathrm{C}$. The thermal profiles also remained the same, except for the change in the onset of degradation from 1 day to 1 week. This change can be contributed to residual solvent in the film for the 1 day sample, but the 1 week sample had ample time for residual solvent to evaporate from the film and extend the onset of degradation. Samples from 1 week to 7 weeks all show the same onset and thermal profile. This indicates that the films are stable at room temperature and only upon heating does the acid become mobile and have sufficient diffusion in the film to catalyze decomposition.

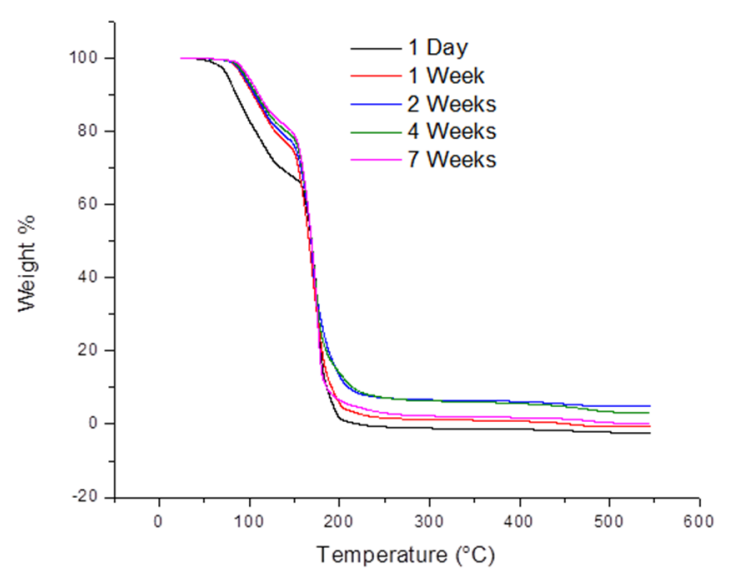

Figure 3. Stability study of the 70/30 PPC/VPC blend + TAG. Thermogravimetric analysis of solution casted films after varying amounts of time at room temperature.

The polymer nanocomposites of PPC + TAG + OMMT and the blend + TAG + OMMT were analyzed to determine the effect of the addition of OMMT on the thermal properties of the systems. OMMT was added as a reinforcing phase to the flexible PPC-based polymers to provide a series of substrates with enhanced mechanical properties. The corresponding mechanical 
analysis for the samples is described below. As the amount of OMMT was increased from $2 \mathrm{wt} \%$ to $10 \mathrm{wt} \%$, the $\mathrm{T}_{\mathrm{d} \text {,onset }}$ and the $\mathrm{T}_{\mathrm{d}, 50}$ increased as seen in Figure 4. For PPC $+\mathrm{TAG}+\mathrm{OMMT}$ (Figure 4A), the addition of $10 \mathrm{wt} \%$ OMMT increased the $\mathrm{T}_{\mathrm{d}, 50}$ from $146{ }^{\circ} \mathrm{C}$ to $203{ }^{\circ} \mathrm{C}$, and for the blend $+\mathrm{TAG}+$ OMMT (Figure 4B), there was a $99{ }^{\circ} \mathrm{C}$ increase in $\mathrm{T}_{\mathrm{d}, 50}$ from $165^{\circ} \mathrm{C}$ to 264 ${ }^{\circ} \mathrm{C}$ with the addition of $10 \mathrm{wt} \%$ OMMT. With 2 and $5 \mathrm{wt} \%$ OMMT added there was less of an increase in the decomposition temperature. One possible explanation for the increase in decomposition temperature with the addition of OMMT is that the when MMT is modified to OMMT there are added surfactants which could interfere with the protonation and degradation of the polymer. Another possible explanation is that the OMMT acts as a diffusion barrier for the TAG resulting in an increase in the decomposition time and temperature. To help mitigate the increase in decomposition temperature from the OMMT, the amount of TAG can be increased from $5 \mathrm{wt} \%$ to $10 \mathrm{wt} \%$ (while keeping the amount of OMMT constant). This helps lower the decomposition temperature $\left(\mathrm{T}_{\mathrm{d}, 50}\right)$ by $52{ }^{\circ} \mathrm{C}$, as seen in Figure $4 \mathrm{C}$ from 211 to $159{ }^{\circ} \mathrm{C}$ going from 5 to $10 \mathrm{wt} \%$ TAG. The addition of OMMT or a clay material introduces a non-degradable component to the polymer systems. This can be seen in the thermal profiles, where the weight fraction remaining at the end of the heating ramp corresponds to the amount of OMMT added (2, 5 , or $10 \mathrm{wt} \%$ ) for both PPC and the blend. However, OMMT particles are between $2-13 \mu \mathrm{m}$ in their longest dimension, which is considered invisible to the naked eye, meaning the remaining OMMT left behind after thermal degradation is unidentifiable and in a dust-like form.

In all cases the thermal evaluation of the polymer materials showed a steep slope (when plotting weight $\%$ vs temperature) once degradation was triggered, indicating fast decomposition of the material. Both VPC and PPC have a single step degradation, which occurs within minutes of degradation starting and the blend polymer has a two step degradation, one step from the VPC component and one step from the PPC component, which have some overlap. Even with the addition of OMMT to PPC and the blend, the resulting decomposition using TGA showed a steep degradation slope indicating that once started, degradation proceeds quickly. The results shown for the various samples were measured at a heating rate of $10^{\circ} \mathrm{C} / \mathrm{min}$, but samples were tested at various heating rates, and over a range of heating rates (between 2 and $20^{\circ} \mathrm{C} / \mathrm{min}$ ), there was no detectable difference in the degradation behavior. The thermal degradation in these systems provides a fast and clean (less than $5 \mathrm{wt} \%$ remaining) method of triggered transience. 

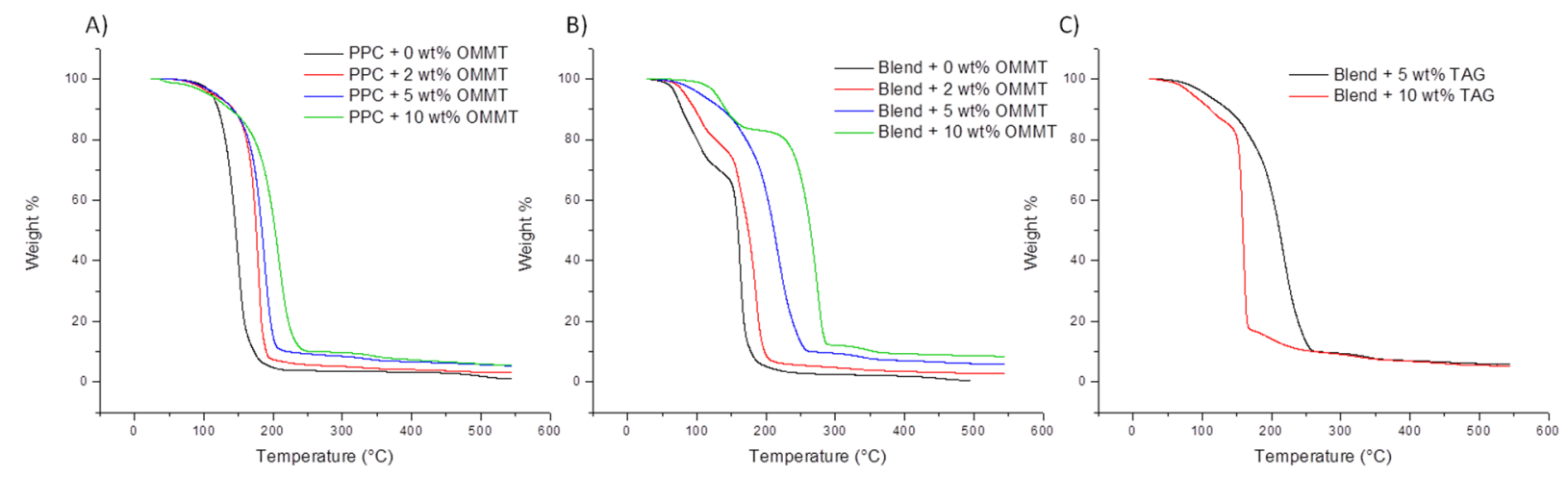

D)

\begin{tabular}{ccc}
\hline Sample & Onset $\mathrm{T}_{\mathrm{d}}\left({ }^{\circ} \mathrm{C}\right)$ & $50 \mathrm{wt} \% \mathrm{~T}_{\mathrm{d}}\left({ }^{\circ} \mathrm{C}\right)$ \\
\hline PPC + TAG & 109 & 146 \\
PPC + TAG + 2 wt\% OMMT & 92 & 176 \\
PPC + TAG + 5 wt\% OMMT & 102 & 184 \\
PPC + TAG + 10 wt\% OMMT & 87 & 203 \\
Blend + TAG & 62 & 165 \\
Blend + TAG + 2 wt\% OMMT & 70 & 174 \\
Blend + TAG + 5 wt\% OMMT & 82 & 211 \\
Blend + TAG + 10 wt\% OMMT & 114 & 264 \\
\hline
\end{tabular}

Figure 4. Thermal analysis of PPC and PPC/VPC 70/30 blend with TAG and OMMT. Thermogravimetric curves of PPC (A) and the blend (B) with $5 \mathrm{wt} \%$ TAG and varying amounts of OMMT, and of the blend with $5 \mathrm{wt} \%$ OMMT and varying amounts of TAG (C). (D) Summary of $\mathrm{T}_{\mathrm{d}, \text { onset }}$ and $\mathrm{T}_{\mathrm{d}, 50}$ as a function of OMMT content of PPC and the blend with varying amounts of OMMT..

For fast and clean decomposition of a thermally triggered transient material, the polymers should produce volatile products. PPC and polycarbonates similar to VPC have been reported to produce volatile decomposition products $^{21,23}$; therefore GC-MS was used to identify the thermolysis products of both VPC and PPC to understand if similar decomposition pathways were followed. A film blend of 70/30 PPC/VPC (no other additives) was solution cast and used for decomposition product analysis since when the blend decomposes it will contain products from both VPC and PPC and both can be measured simultaneously. A small piece of the film was placed in a sealed vial and heated to decomposition. After cooling a small amount of DCM was added to the vial and shaken to dissolve any products. The solution was then analyzed using a GC-MS. The predicted products from thermal decomposition of PPC and VPC can be seen in 
Scheme 1. The resulting analysis of the products using GM-MS confirms these predicted products and the analysis of the mass peaks can be seen in Table 1 . The peaks corresponding to the parent molecule and its resulting fragmentation peaks were identified for each degradation product.

Scheme 1. Major thermal degradation products of VPC (a) and PPC (b).

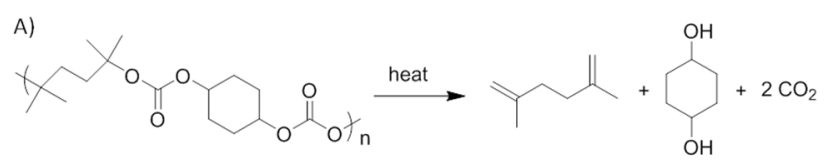

B)

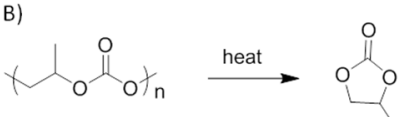

Table 1. GC-MS analysis of the thermal degradation products of VPC and PPC.

\begin{tabular}{|c|c|c|}
\hline Polymer & Fragment & $\begin{array}{c}\text { Characteristic peaks } \\
(\mathrm{m} / \mathrm{z})\end{array}$ \\
\hline VPC & $\begin{array}{c}\text { 2,5-dimethyl-1,5- } \\
\text { hexadiene }\end{array}$ & $110,95,84,73,67,56$ \\
\hline & 1,4-cyclohexandiol & $98,83,69,55,46$ \\
\hline PPC & Propylene carbonate & $102,87,58,45$ \\
\hline
\end{tabular}

\section{Mechanical Analysis}

The Young's moduli, toughness, and tensile strength of the PPC + OMMT and the PPC/VPC 70/30 blend + OMMT composites were determined using DMA. As mentioned above the VPC alone did not form stable thick films and, therefore, DMA testing could not be conducted. The solution cast composites were peeled from their PDMS substrates and cut into $3.5 \mathrm{~mm}$ strips. Each sample was mounted and subjected to a constant force ramp of $1 \mathrm{~N} / \mathrm{min}$ at room temperature until the sample yielded, producing a stress vs strain curve. The Young's modulus for each sample was determined from the linear portion during the initial strain $(0.2-$ $0.4 \%$ strain) and can be seen in Figure 5. In the PPC + OMMT composite (Figure 5A and C), as the amount of OMMT added increased the modulus also increased, accessing a range of moduli from $2 \mathrm{MPa}$ with no OMMT to $1.3 \mathrm{GPa}$ with $10 \mathrm{wt} \%$ OMMT. The blend + OMMT composite showed a similar trend, with an increase in OMMT leading to an increase in modulus to a certain extent as seen in Figure 5B and D. The addition of VPC to the PPC/VPC blend already helps improve the modulus from $2 \mathrm{MPa}$ to $105 \mathrm{MPa}$, and the further addition of OMMT increases the 
modulus to a maximum of $1.9 \mathrm{GPa}$. A cross-sectional SEM image of the PPC/VPC blend with 10 $\mathrm{wt} \%$ OMMT can be seen in Figure 6. Figure 6A shows the OMMT is spread throughout the film, with some settling of the OMMT to the bottom which can be attributed to the solution casting and subsequent drying process, and is consistent throughout the different loadings of OMMT. At $10 \mathrm{wt} \%$ OMMT added in the blended system there is a small amount of aggregation which was not seen in the other samples. This aggregation could lead to the decrease in modulus from the 5 to $10 \mathrm{wt} \%$ added OMMT. In all samples it can be seen that the OMMT sheets are randomly oriented, but despite this they are still able to provide mechanical improvements to the film. The OMMT sheets are microns in length and nanometers in width which allows them to operate in both a nano- and micro-meter regime. The dual regime as well as compatibility with the polymer system provides improvement in the Young's modulus at low loadings ${ }^{25-27}$.

The toughness of the polymers and their composites can be determined by finding the area under the curve in a stress vs strain plot, and can be seen in Figure 5A and C for PPC and Figure $5 \mathrm{~B}$ and $\mathrm{D}$ for the blend. As the amount of OMMT increases the toughness of the composites decreases, meaning they absorb less energy before failure. The OMMT adds a brittle component to the PPC and the blend, which improves their modulus but makes them less fracture resistant. The toughness decreases by two orders of magnitude for PPC when $10 \mathrm{wt} \%$ OMMT is added, and decreases by one order of magnitude for the blend when $5 \mathrm{wt} \%$ OMMT is in the composite. The tensile strength of the films can also be determined from the stress vs strain curve. The tensile strength is the maximum stress value the film achieves before failure. Tensile strength values for the films tested can be seen in Figure 5B and D for PPC + OMMT and blend + OMMT, respectively. As the amount of OMMT is increased, the tensile strength also increases. PPC alone had a measured tensile strength of $1.2 \mathrm{MPa}$, and the addition of $10 \mathrm{wt} \%$ OMMT increased the tensile strength to 7.2 MPa. The same trend was observed for the blend, with the blend alone having a tensile strength of $4.8 \mathrm{MPa}$ and increased to $15 \mathrm{MPa}$ with the addition of $10 \mathrm{wt} \%$ OMMT.

The addition of OMMT allows for a range of mechanical properties to be achieved, allowing for the system to be used across various applications from flexible substrates to more rigid substrates. For applications in transient electronics, it is important to have materials with a higher Young's modulus (close to $1 \mathrm{GPa}$ or better) to provide a structural support for batteries, 
fuel cells, and other electronic components. Maintaining a moderate toughness is important so your substrate doesn't fracture due to operation of the device.
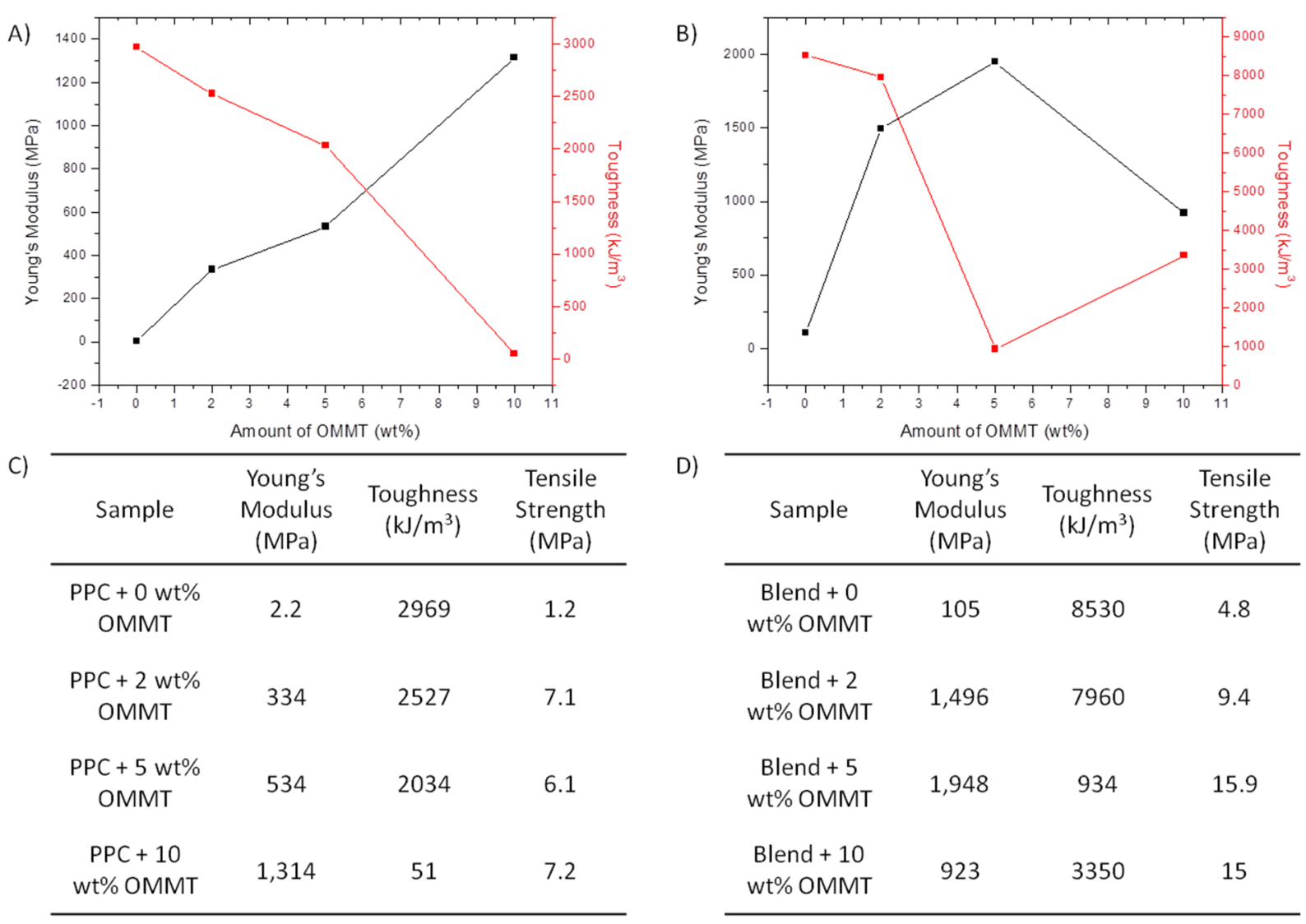

D)

\begin{tabular}{cccc}
\hline Sample & $\begin{array}{c}\text { Young's } \\
\text { Modulus } \\
(\mathrm{MPa})\end{array}$ & $\begin{array}{c}\text { Toughness } \\
\left(\mathrm{kJ} / \mathrm{m}^{3}\right)\end{array}$ & $\begin{array}{c}\text { Tensile } \\
\text { Strength } \\
(\mathrm{MPa})\end{array}$ \\
\hline $\begin{array}{c}\text { Blend + 0 } \\
\text { wt\% OMMT }\end{array}$ & 105 & 8530 & 4.8 \\
$\begin{array}{c}\text { Blend + } 2 \\
\text { tt\% OMMT }\end{array}$ & 1,496 & 7960 & 9.4 \\
$\begin{array}{c}\text { Blend + 5 } \\
\text { wt\% OMMT }\end{array}$ & 1,948 & 934 & 15.9 \\
$\begin{array}{c}\text { Blend + 10 } \\
\text { wt\% OMMT }\end{array}$ & 923 & 3350 & 15 \\
\hline
\end{tabular}

Figure 5. Mechanical properties of PPC with various amounts of OMMT and the blend with various amounts of OMMT. The Young's Modulus and toughness are plotted for comparison for PPC (A) and blend (B), and the values for modulus, toughness, and tensile strength are listed for PPC (C) and blend (D). 

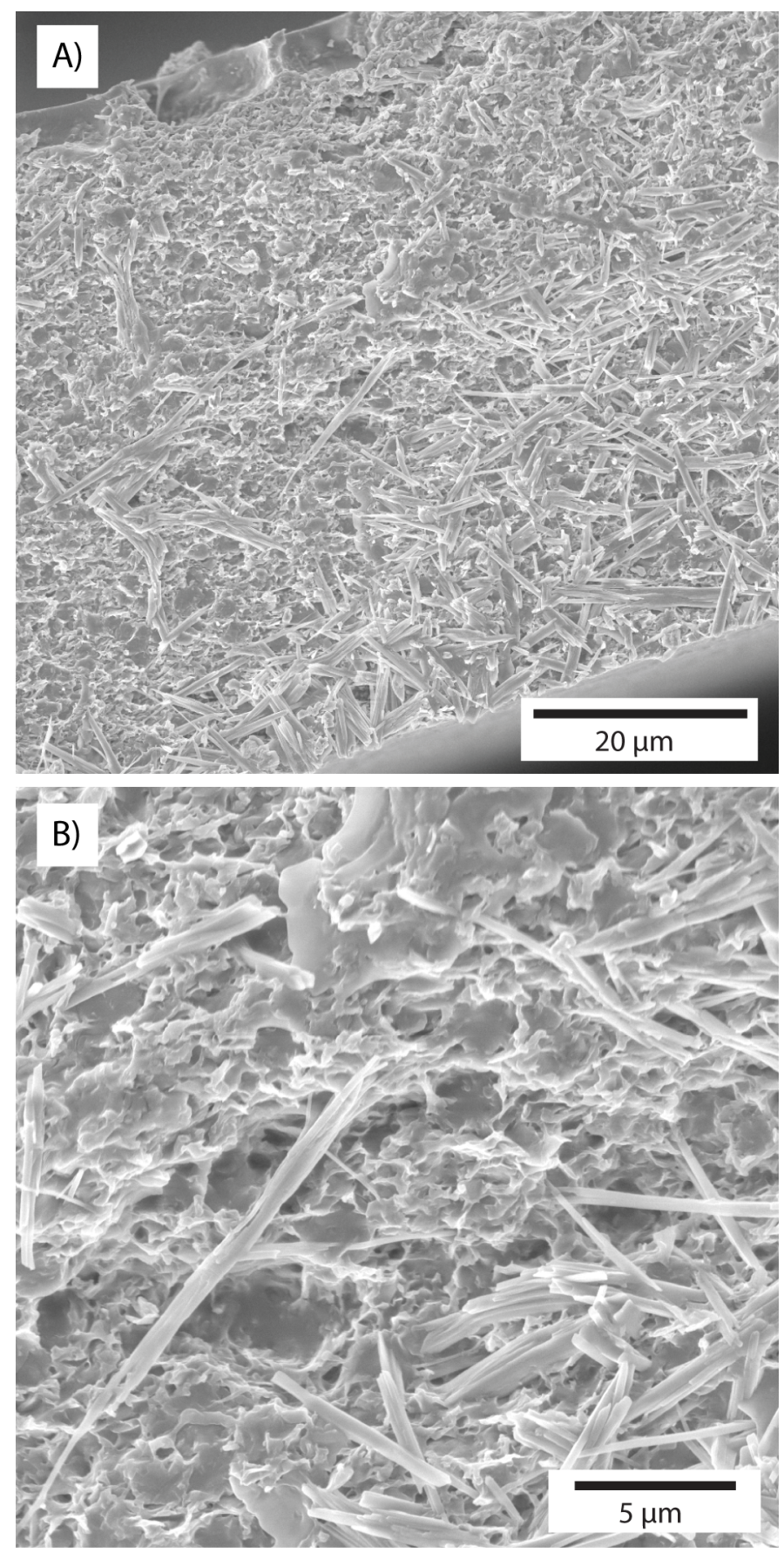

Figure 6. Cross-sectional SEM of 70/30 PPC/VPC with $10 \mathrm{wt} \%$ OMMT (a) and a zoomed in section (b).

\section{Conclusion:}

Multiple polycarbonate systems were analyzed to determine the effect of acid additives, compositing, and blending on the thermal and mechanical properties of the system. It was 
determined that films of varying thickness $(100 \mathrm{~nm}-300 \mu \mathrm{m})$ can be formed from VPC and PPC, as well as a blend of the two polycarbonates. VPC and PPC, as well as the blend, were found to be acid sensitive and showed catalyzed decomposition with both a PAG and TAG addition. The $\mathrm{T}_{\mathrm{d}, 50}$ was lowered by $\sim 70{ }^{\circ} \mathrm{C}$ and the films were stable at room temperature with TAG for up to 7 weeks. The addition of TAG allows for the blended polymer system to achieve lower degradation temperatures than with PAG, and the blended system provides the ability to form thick substrates. Both PPC and the blend had a fairly low modulus and are flexible materials, which is not beneficial in some applications. The addition of OMMT increased the modulus of the materials, but also decreased the toughness. Depending on application, one can adjust the amount of OMMT needed to achieve the desired mechanical properties. With addition of OMMT also comes an increase in the degradation temperatures of the materials, which can be mitigated by the increasing the amount of acid in the system.

The thermal catalysis and tunable mechanical properties allows for the use of these polymers as thermally sensitive substrates in packaging for transient materials. These polymer substrates are stable at room temperature, but once heat is applied at mild temperatures the substrate degrades within minutes, allowing for a triggered transience to occur. One can simply apply heat to the substrate and have it disappear after it has performed its desired function.

\section{Acknowledgements:}

This research was primarily supported by the Defense Advanced Research Project Agency (DARPA) and the Air Force Research Laboratory (AFRL) award FA8650-14-C7402. This work made use of the Cornell Center for Materials Research Shared Facilities which are supported through the NSF MRSEC program (DMR-1120296). This work was performed in part at the Cornell NanoScale Facility, a member of the National Nanotechnology Coordinated Infrastructure (NNCI), which is supported by the National Science Foundation (Grant ECCS15420819). The authors would like to thank Serhan Ardanuc, Ved Gund, Alexander Ruyack, Jeff Kriz, Steven Tin, Jeff Ridley, and Steven Eickhoff for their fruitful discussions on the topic. 


\section{References:}

1. Harnett CK, Coates GW, Craighead HG. Heat-depolymerizable polycarbonates as electron beam patternable sacrificial layers for nanofluidics. J Vac Sci Technol B Microelectron Nanom Struct. 2001;19(6):2842. doi:10.1116/1.1409383.

2. Reed HA, White CE, Rao V, Allen SAB, Henderson CL, Kohl PA. Fabrication of microchannels using polycarbonates as sacrificial materials. J Micromechanics Microengineering. 2001;11(6):733-737. doi:10.1088/0960-1317/11/6/317.

3. Jayachandran JP, Reed H a., Zhen H, et al. Air-channel fabrication for microelectromechanical systems via sacrificial photosensitive polycarbonates. $J$ Microelectromechanical Syst. 2003;12(2):147-159. doi:10.1109/JMEMS.2003.809963.

4. Loo LS, Gleason KK. Hot Filament Chemical Vapor Deposition of Polyoxymethylene as a Sacrificial Layer for Fabricating Air Gaps. Electrochem Solid-State Lett. 2001;4(11):G81. doi:10.1149/1.1402496.

5. Li W, Tegenfeldt JO, Chen L, et al. Sacrificial polymers for nanofluidic channels in biological applications. Nanotechnology. 2003;14(6):578-583. doi:10.1088/09574484/14/6/302.

6. Thanoo BC, Sunny MC, Jayakrishnan A. Oral Sustained-release Drug Delivery Systems using Polycarbonate Microspheres Capable of Floating on the Gastric Fluid. J Pharm Pharmacol. 1993;45(1):21-24. doi:10.1111/j.2042-7158.1993.tb03672.x.

7. Joseph NJ, Lakshmi S, Jayakrishnan A. A floating-type oral dosage form for piroxicam based on hollow polycarbonate microspheres: in vitro and in vivo evaluation in rabbits. $J$ Control Release. 2002;79(1-3):71-79. doi:10.1016/S0168-3659(01)00507-7.

8. Chen W, Meng F, Li F, Ji SJ, Zhong Z. pH-responsive biodegradable micelles based on acid-labile polycarbonate hydrophobe: Synthesis and triggered drug release. Biomacromolecules. 2009;10(7):1727-1735. doi:10.1021/bm900074d.

9. Makadia HK, Siegel SJ. Poly Lactic-co-Glycolic Acid (PLGA) as Biodegradable Controlled Drug Delivery Carrier. Polymers (Basel). 2011;3(4):1377-1397. doi:10.3390/polym3031377.

10. Ponnusamy T, Lawson LB, Freytag LC, Blake DA, Ayyala RS, John VT. In vitro degradation and release characteristics of spin coated thin films of PLGA with a "breath figure" morphology. Biomatter. 2012;2(2):77-86. doi:10.4161/biom.20390.

11. Hwang S-W, Tao H, Kim D-H, et al. A Physically Transient Form of Silicon Electronics. Science (80- ). 2012;337(6102):1640-1644. doi:10.1126/science.1226325.

12. Hernandez HL, Kang S-K, Lee OP, et al. Triggered Transience of Metastable Poly(phthalaldehyde) for Transient Electronics. Adv Mater. 2014;26(45):7637-7642. doi:10.1002/adma.201403045.

13. Yin L, Cheng H, Mao S, et al. Dissolvable metals for transient electronics. Adv Funct Mater. 2014;24(5):645-658. doi:10.1002/adfm.201301847.

14. Hwang S-W, Song J-K, Huang X, et al. High-Performance Biodegradable/Transient 
Electronics on Biodegradable Polymers. Adv Mater. 2014;26(23):3905-3911. doi:10.1002/adma.201306050.

15. Acar H, Çınar S, Thunga M, Kessler MR, Hashemi N, Montazami R. Study of Physically Transient Insulating Materials as a Potential Platform for Transient Electronics and Bioelectronics. Adv Funct Mater. 2014;24(26):4135-4143. doi:10.1002/adfm.201304186.

16. Kang SK, Hwang SW, Cheng H, et al. Dissolution behaviors and applications of silicon oxides and nitrides in transient electronics. Adv Funct Mater. 2014;24:4427-4434. doi:10.1002/adfm.201304293.

17. Park CW, Kang S-K, Hernandez HL, et al. Thermally Triggered Degradation of Transient Electronic Devices. Adv Mater. 2015;27(25):3783-3788. doi:10.1002/adma.201501180.

18. Fréchet JMJ, Eichler E, Stanciulescu M, et al. Chapter 12 Acid-Catalyzed Thermolytic Depolymerization of Polycarbonates : A New A p p r o a c h to Dry-Developing Resist Materials The design of $\mathrm{n}$ e $\mathrm{w}$ condensation polymers which undergo thermolysis is explored with polycarbonates . The polymers are prep. 1987:138-148.

19. Yu A, Liu H, Blinco JP, et al. Patterning of tailored polycarbonate based non-chemically amplified resists using extreme ultraviolet lithography. Macromol Rapid Commun. 2010;31(16):1449-1455. doi:10.1002/marc.201000117.

20. White CE, Balogun A, Henderson CL. Effects of the Photoacid Generator Type on the Imaging and Thermal Decomposition Properties of Photodefinable, Thermally Sacrificial Poly ( propylene carbonate ) Materials. J Appl Polym Sci. 2006;102:266-271. doi:10.1002/app.23724.

21. Li XH, Meng YZ, Zhu Q, Tjong SC. Thermal decomposition characteristics of poly(propylene carbonate) using TG/IR and Py-GC/MS techniques. Polym Degrad Stab. 2003;81(1):157-165. doi:10.1016/S0141-3910(03)00085-5.

22. Spencer TJ, Kohl P a. Decomposition of poly(propylene carbonate) with UV sensitive iodonium salts. Polym Degrad Stab. 2011;96(4):686-702. doi:10.1016/j.polymdegradstab.2010.12.003.

23. Houlihan FM, Bouchard F, Fréchet JMJ, Willson CG. Thermally depolymerizable polycarbonates. 2. Synthesis of novel linear tertiary copolycarbonates by phase-transfer catalysis. Macromolecules. 1986;19(1):13-19. doi:10.1021/ma00155a003.

24. Shi X, Gan Z. Preparation and characterization of poly(propylene carbonate)/montmorillonite nanocomposites by solution intercalation. Eur Polym J. 2007;43(12):4852-4858. doi:10.1016/j.eurpolymj.2007.09.024.

25. Giannelis EP. Polymer Layered Silicate Nanocomposites. Adv Mater. 2004;8(1):29-35. doi:10.1002/adma.19960080104.

26. Alexandre M, Dubois P. Polymer layered-silicate nanocomposites: preparation, properties and uses of a new class of materials. Mater Sci Eng. 2000;28(March):1-63. doi:10.1016/S0927-796X(00)00012-7.

27. Lebaron PC, Wang Z, Pinnavaia TJ. Polymer-layered silicate nanocomposites: An overview. Appl Clay Sci. 1999;15(1-2):11-29. doi:10.1016/S0169-1317(99)00017-4. 